\title{
Concurrent Chagas' disease and borderline disseminated cutaneous leishmaniasis: The role of amiodarone as an antitrypanosomatidae drug
}

\author{
Alberto E Paniz-Mondolfi ${ }^{1,4}$ \\ Alexandra M Pérez-Álvarez' \\ Oscar Reyes-Jaimes² \\ Gustavo Socorro' \\ Olga Zerpa ${ }^{3}$ \\ Denisa Slova ${ }^{4}$ \\ Juan L Concepción ${ }^{5}$

\begin{abstract}
'Laboratorio de Estudio de Antígenos, Instituto de Biomedicina, "Hospital José Gregório Hernández", Instituto Venezolano de los Seguros Sociales, Caracas, Venezuela; ${ }^{2}$ Departamento de Dermopatología, Instituto de Biomedicina, ${ }^{3}$ Sección de Leishmaniasis, Instituto de Biomedicina, Caracas, Venezuela; ${ }^{4}$ Department of Pathology and Laboratory Medicine, St. Luke's Roosevelt - Beth Israel Medical Center, University Hospital of Columbia University College of Physicians and Surgeons, New York, USA; ${ }^{5}$ Laboratorio de Enzimología de Parásitos, Facultad de Ciencias, Universidad de Los Andes, Mérida, Venezuela
\end{abstract}

\begin{abstract}
The occurrence of mixed infections of Trypanosoma cruzi and Leishmania spp. is becoming a common feature in Central and South America due to overlapping endemic areas. Unfortunately, the possibilities for treating flagellated kinetoplastid infections are still very limited and most of the available drugs exhibit severe side effects. Although the development of new drugs for Leishmania has markedly improved in the last years, the tendency is still to employ antimonial compounds. On the other hand, treatment for Chagas' disease is only available for the acute phase with no effective therapeutical options for chronic stage disease. The following case report substantiates the recently discovered effect of amiodarone as a nonconventional antiparasitic drug, particularly against Leishmania, breaching a new perspective in the therapeutic management of these important infectious parasitic diseases.
\end{abstract}

Keywords: Chagas' disease, leishmaniasis, chemotherapy, amiodarone

\section{Introduction}

Leishmania and Trypanosoma are digenetic flagellated kinetoplastid protozoa implicated as the causative agents of two of the most important parasitic endemic diseases in Central and South America: Leishmaniasis and Chagas' disease, respectively (Mendes et al 2007). American cutaneous leishmaniasis comprises a spectrum of clinical syndromes characterized by two polar forms: localized cutaneous leishmaniasis, which is observed in patients which develop a competent immune response against the parasite, and diffuse cutaneous leishmaniasis characterized by extensive lesions and a specific $\mathrm{T}$ cell immunodeficiency in the host (Convit 1996). Intermediate forms of the disease (mucocutaneous and borderline disseminated cutaneous leishmaniasis) are rare entities that often reflect a partial immunodeficiency with an exaggerated or even absent delayed type hypersensitivity respectively (Convit et al 1993; Silveira 2004). On the other hand, American trypanosomiasis or Chagas' disease caused by the hemoflagellate protozoa Trypanosoma cruzi, is a biphasic disease that exhibits an acute as well as a chronic phase, the latter with severe and fatal complications such as chagasic cardiomyopathy (Dias et al 1956).

Conventional therapy of leishmaniasis comprises the utilization of pentavalent antimonials demanding a careful monitoring of the renal and liver function, so as a previous cardiac evaluation that must include an electrocardiographic study due to the known cardiotoxicity of antimonials (Murray et al 2005; Schwartz 2006). For Chagas' disease, current treatment of the acute phase is based on nitrofurans (nifurtimox) and nitroimidazoles (benznidazole), nevertheless, these compounds have proven to be of limited efficacy in chronic stages and their use is restricted because of their important toxic side effects (Urbina and Docampo 2003). Systematic reviews of controlled trials including asymptomatic and symptomatic patients have revealed that there is insufficient data to 
provide evidence that currently available trypanosomatidal therapy or any other drug used for the symptomatic treatment of chagasic cardiomyopathy can modify clinical outcomes in chronic Chagas disease (Marin-Neto et al 2007). However, nowadays the development of new drugs and studies on the anti-trypanosomatidal effect of some old compounds such as amiodarone, have opened new insights in the therapeutical approach and management of these parasitic diseases.

The following case reveals the potential utility of amiodarone, not only for the symptomatic treatment of arrhytmias in Chagas' disease and its possible anti-parasitic effect against T. cruzi, but, in the treatment of other kinetoplastid protozoal infections such as leishmaniasis.

\section{Case report}

A 74 year-old male agriculture worker from the central western region of the Venezuelan plains (Llanos) was transferred to our center for further management of his cutaneous condition. The infection had started one year previous to his admission with a primary skin lesion in the form of a firm erythemathous nodule that evolved to an irregularly shaped infiltrated plaque located in his left lower extremity. After two months, the plaque ulcerated with the concomitant appearance of multiple small nodular metastatic lesions.

Upon admission and initial evaluation, he was found to have a $7 \times 5 \times 4.5 \mathrm{~cm}$ ulcer with heavily infiltrated borders and multiple satellite infiltrated nodular and acneiform lesions measuring $2 \mathrm{~cm}$ in diameter with the largest located at only $5 \mathrm{~cm}$ from the primary ulcerated lesion (Figures 1 and 2). A Giemsa-stained skin smear revealed the presence of few amastigotes in the lesions (Figure 3) and delayed type hypersensitivity (DTH) skin-test was negative. Histological sections of the lesions revealed a vacuolated granuloma exhibiting large collections of vacuolated and heavily parasitized macrophages surrounded by a lympho plasmacytic infiltrate (Figure 4). Further parasite culture and typification confirmed the presence of Leishmania (Viannia) braziliensis as the etiologic agent.

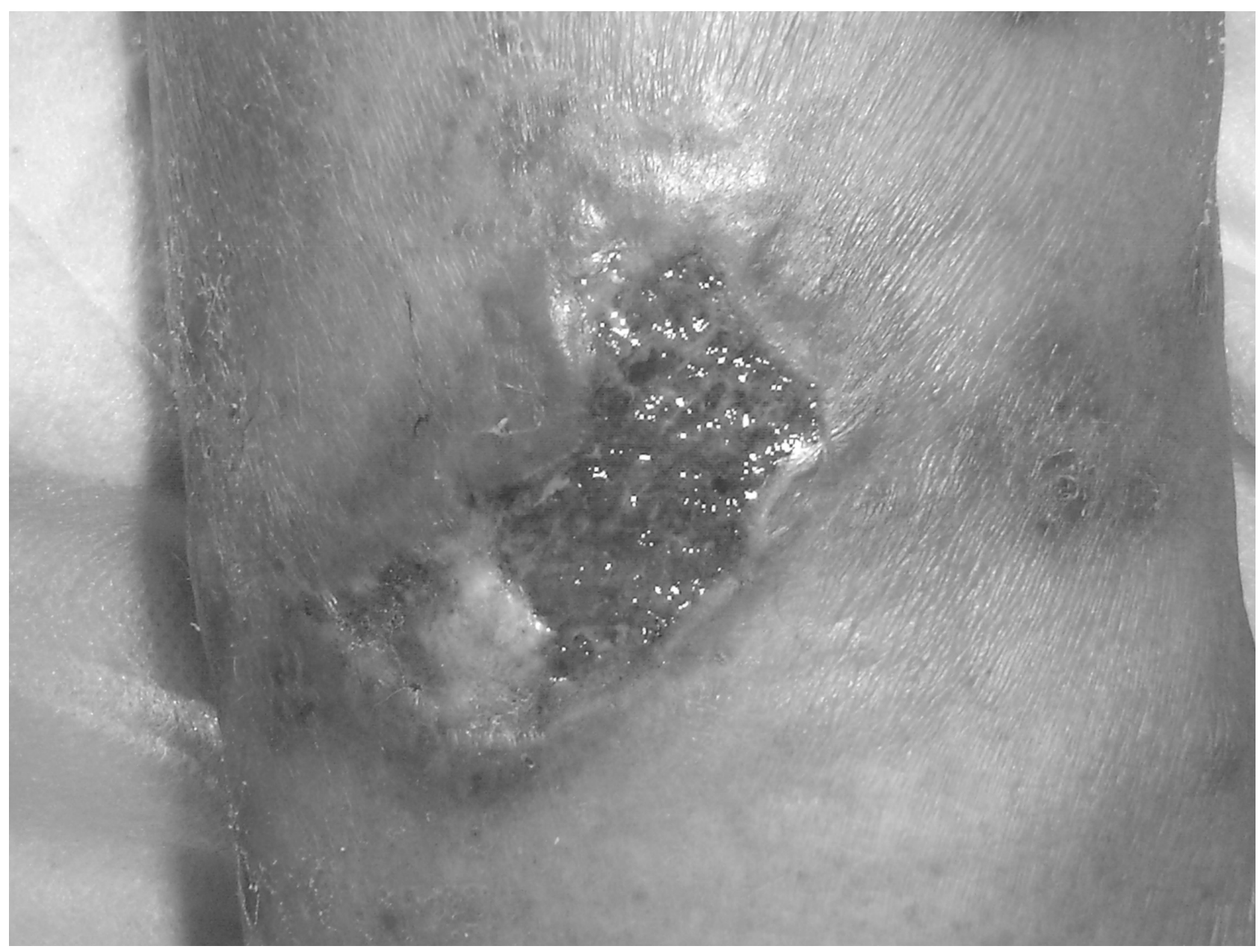

Figure I Ulcerated lesion with partial re-epithelization after the tenth day of treatment. 


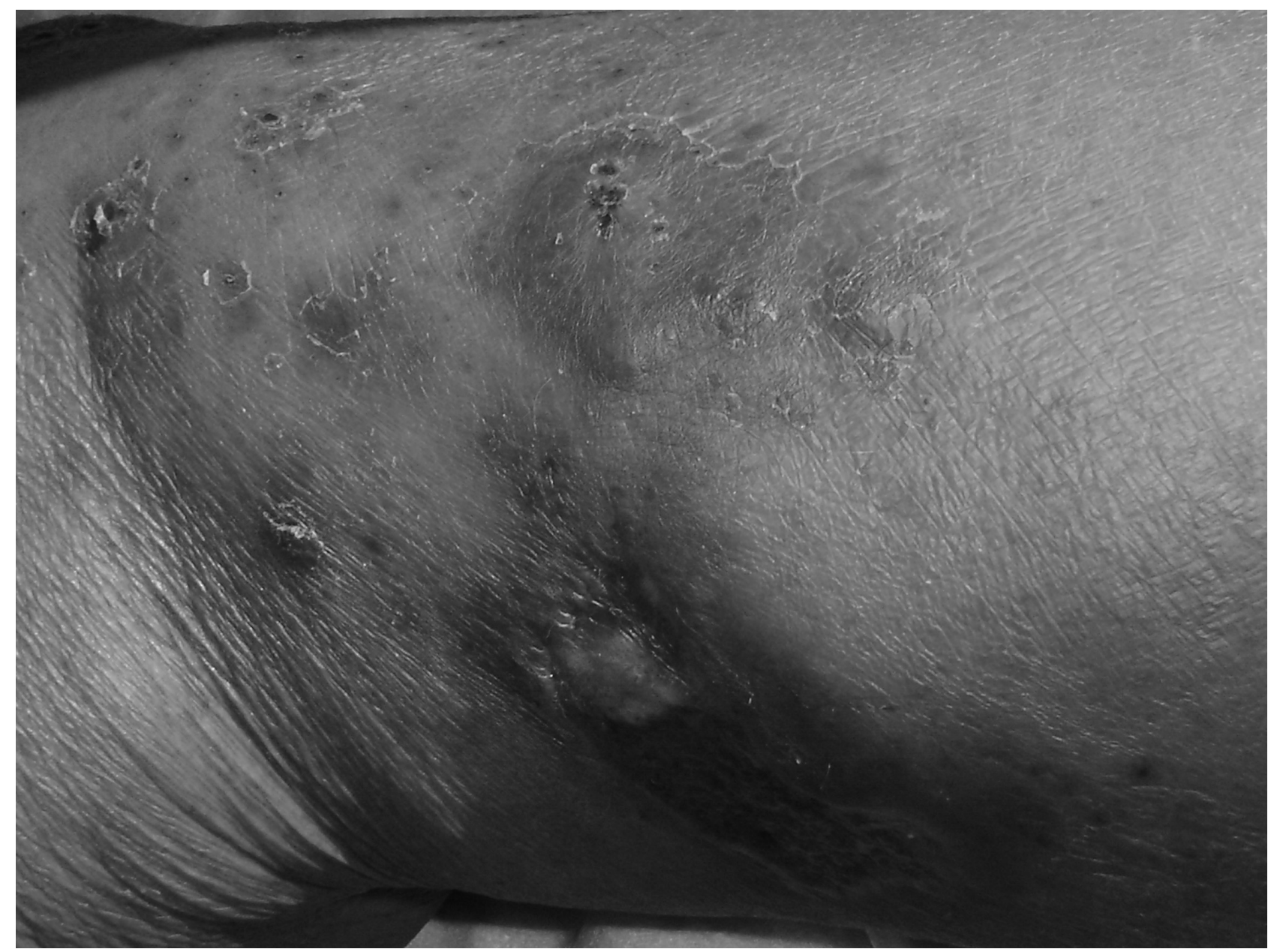

Figure 2 Satellite disseminated acneiform lesions with moderate perilesional infiltration.

The past medical history of the patient was otherwise unremarkable, except for episodes of dispnea, ortopnea, and palpitations over the last three months. The physical examination revealed jugular vein distention, prominent audible $\mathrm{S} 3$ heart sound, and a laterally displaced cardiac apex; rales were audible in both lung bases and mild pitting edema was evident in lower extremities; the rest of the physical examination was unremarkable. A complete cardiovascular workup was initiated to asses the cause of the symptoms. The chest radiography revealed cardiomegaly and discrete bilateral pleural effusions. On echocardiography focal segmental abnormalities were evident mainly in the posterioinferior aspect of the left ventricle with moderate hypokinesia. A conventional 12-lead electrocardiogram (EKG) and a 24-hour Holter monitoring demonstrated the presence of a non-sustained ventricular tachycardia and ventricular extrasystoles.

Complete blood count was unremarkable, chemistry panel revealed: urea nitrogen: $21.0 \mathrm{mg} / \mathrm{dl}$, creatinine: $2.3 \mathrm{mg} / \mathrm{dl}$, sodium: $136 \mathrm{mEq} / \mathrm{L}$, potassium: $4.0 \mathrm{mEq} / \mathrm{L}$. Because the patient came from a highly endemic area of Chagas' disease he was tested for this illness, resulting positive in the Machado-Guerreiro complement-fixation test which was then confirmed by enzyme-linked immunosorbent assay with recombinant highly specific antigens.

Due to his underlying cardiovascular condition the possibility of initiating pentavalent antimonial therapy for Leishmania was discarded temporarily until stabilization, and the patient was immediately initiated on Amiodarone with a loading regimen of $1600 \mathrm{mg}$ daily for the first four days and a further maintenance dose of $800 \mathrm{mg}$ /day for three weeks; adequate control of the ventricular arrhytmyas was achieved, and patient's CHF condition improved with supportive symptomatic treatment. Strikingly, the cutaneous lesions also started to improve despite the absence of specific antileishmanial therapy; the ulcers started to re-epithelize since the fourth day, and after fifteen days parasitic forms were not found on microscopic examination of control smears and biopsies. Patient was discharged from our center and 


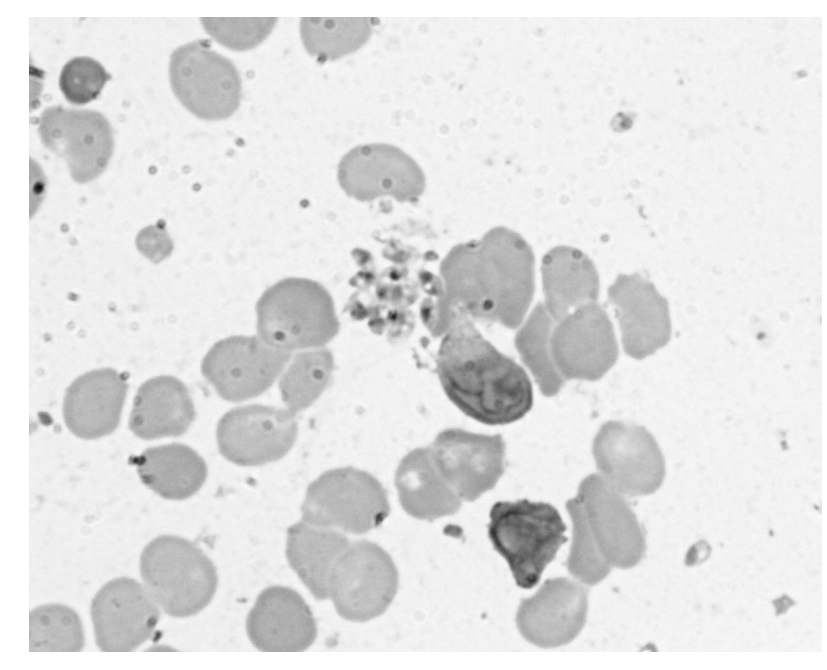

Figure 3 Giemsa-stained skin smear revealing parasites disposed in groups, note the typical round nucleus and rod-shaped kinetoplast in the body of the amastigotes.

remitted to the corresponding dermatology clinic in his region who informed us that the ulcer had completely healed after one month and that after one year no new lesions developed with serially negative control biopsies (read in our laboratory) declaring the clinical cure of this patient.

Spirometry testing and thyroid function tests were performed at the beginning and after the period of treatment remaining under normal limits. The patient was remitted to a cardiology center for further follow up of his Chagas' disease continuing his symptomatic treatment and a low dose ( $200 \mathrm{mg}$ /day) regimen of amiodarone for prevention of arrhytmias, with a notable reduction in his congestive heart failure exacerbation episodes (personal communication from the regional sanitary office).

\section{Discussion}

Human mixed infections of Leishmania spp. and T. cruzi are becoming a common clinical feature in Central and South America due to overlapping endemic areas of both diseases (Bastrenta et al 2003). Secondary invasion of the parasite's ecological habitat because of degradation of the local environment, due to the growing population density extending to periurban regions is the main reason of the increment in the number of cases which can initially, and for long periods of time, be asymptomatic (Mendes et al 2007).

Following an initial acute phase in which signs and symptoms are usually nonspecific, Chagas' disease enters a chronic phase that can remain asymptomatic for periods of years or even throughout the lifetime (Dias et al 1956). Most cases, as the one we report, are incidentally diagnosed when consulting for other reasons. Unfortunately, by the time patients with Chagas' disease are diagnosed in the chronic phase, the therapeutical options are of limited effectiveness (Croft et al 2005). In this case the patient had begun to exhibit cardiovascular symptoms which in addition to his epidemiological background led to the suspicion and confirmation of T. cruzi infection with chagasic cardiomyopathy. Nevertheless, this patient consulted specifically because of his cutaneous lesions.

Some authors state that isolated ulcers as occur in cases of localized cutaneous leishmaniasis are usually self-limited and that dangers of treatment should be balanced against the possibility of spontaneous cure (Seear 2000); however in our experience, self-healing lesions are rare, and extremely often are associated to secondary infections that limit the spontaneous healing of the ulcers. On the other hand, cases of borderline disseminated cutaneous leishmaniasis despite aggressive local measures are virtually incurable with out specific chemotherapy and have not been reported in the literature.

After being diagnosed with borderline disseminated cutaneous leishmaniasis, the underlying cardiologic condition of the patient imposed a challenge at the time to treat with pentavalent antimonials due to the associated cardiotoxic effects of these drugs - such as development of ventricular tachyarrhythmias associated with prolongation of the electrocardiographic rate-corrected QT interval (Lawn et al 2006) - which could have potentially aggravated the patient's condition; in this sense the use of antimonials was discarded and no other antileishmanial medication was initiated.

Amiodarone which is a versatile antiarrhytmic agent, chiefly class III but with also strong class I and ancillary class II and IV activity (Opie 2005) was initiated not only to control the patients arrhythmia as part of the symptomatic treatment for his Chagas' disease, but also in an attempt to secondarily

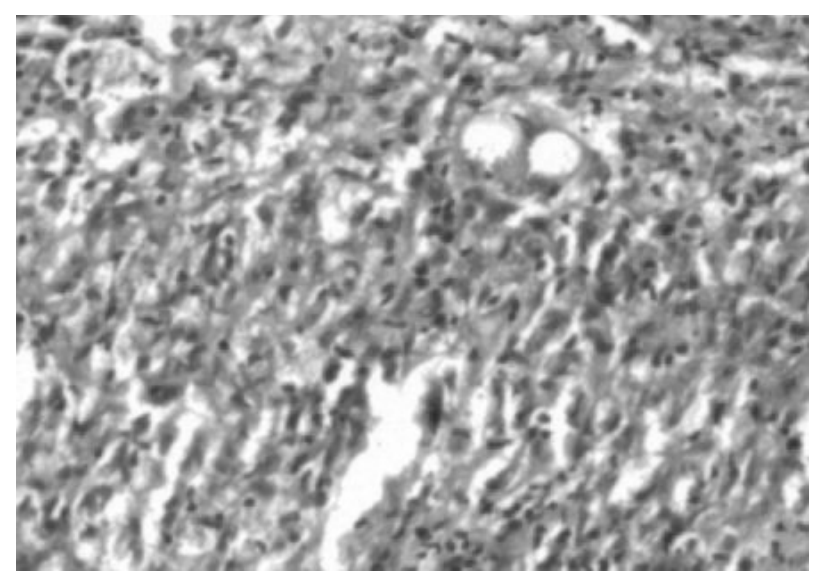

Figure 4 Detail of the histological section standing out the diffuse macrophagic granuloma invaded by numerous lymphoid cells and presence of amastigotes contained in the vacuolated macrophages (Hematoxilin-Eosin x400). 
treat his leishmaniasis, by taking advantage of the recently described properties of this drug as an antiparasitic drug.

The role of amiodarone as an antimicrobial agent was recently revealed by Courchesne (2002) who characterized this compound's broad fungicidal activity by inducing a MID1dependent rise in free cytoplasmic calcium in the baker's yeast Saccharomyces cerevisiae (Courchesene 2003) and by disrupting calcium hemostasis (Gupta 2003). Furthermore its use as an antiprotozoal agent has been newly studied after being proved to be effective against both proliferative stages of T. cruzi in a comparable manner to its antifungal activity (Benaim 2006). Amiodarone acts by interfering with sterol biosynthesis of the parasites membrane and also by inducing a rapid raise in free cytoplasmic calcium levels from intracellular compartments as well as from the mitochondria, provoking a collapse of the mithocondrial membrane potential (Benaim 2006).

On the other hand, the efficacy of amiodarone in Leishmania infection may be favored by its pharmacokinetic characteristics, being the most prominent: its highly lipid-soluble nature, extensive tissue distribution and its important skin excretion (Opie 2005); the latter attribute ensuring its adequate delivery into the site of infection with Leishmania in this patient. Nonetheless, caution has to be exercised when considering treatment with amiodarone because of its restraining side effects such as cardiotoxicity, thyroid disfunction and pulmonary fibrosis (Opie 2005). However, structural modifications in the amiodarone molecule have led to the development of new drugs such as dronedarone, which lack these severe side effects and provide new and more secure therapeutical options (Singh 2006).

Considering that Trypanosoma and Leishmania as related organisms share similar biosynthetic pathways and metabolic processes, it is also possible that amiodarone's anti T. cruzi effects could also be effective against the Leishmania parasite sharing the same underlying molecular mechanisms. The fact that this patient exhibited a clinical parasitological cure under treatment with amiodarone and no other antileishmanial drug sustains this statement and reinforces the efficacy of this chimerical drug as antitrypanosomatidae.

\section{Concluding remarks}

Finally, to our knowledge, this is the first case report in which parasitism by Leishmania is successfully treated with this nonconventional antiparasitic drug; the clinical outcome of this patient appears to indicate that amiodarone exhibits a broad spectrum antiprotozoal activity in addition to its well known antifungal action. Taking into consideration that protozoan pathogens cause an increased morbidity and mortality among millions of humans, especially in developing, tropical, and subtropical countries, the use of amiodarone or its new analogues offer a whole new approach in the treatment of leishmaniasis and other parasitic diseases. Further research on the potential antileishmanial effect of amiodarone on experimental models would be timely in order to reveal the underlying mechanisms of this possible new therapeutic option.

\section{Acknowledgments}

We thank Dr. Luisana Avilán for the critical review of the manuscript. We are most grateful to the reviewers for their precise commentaries on the article.

\section{References}

Bastrenta B, Mita N, Buitrago R, et al. 2003. Human mixed infections of Leishmania spp. And Leishmania-Trypanosoma cruzi in a sub Andean Bolivian area: Identification by polymerase chain reaction/hybridization and isoenzyme. Mem Inst Oswaldo Cruz, 98:255-64.

Benaim G, Sanders JM, Garcia-Marchan Y, et al. 2006. Amiodarone has intrinsic anti-trypanosoma cruzi activity and acts synergistically with posaconazole. J Med Chem, 49:892-99.

Convit J, Ulrich M, Fernandez CT, et al. 1993. The clinical and immunological spectrum of American cutaneous leishmaniasis. Trans Roy Soc Trop Med Hyg, 87:444-8.

Convit J. 1996. Leishmaniasis: Immunological and clinical aspects and vaccines in Venezuela. Clin Dermatol, 14:479-87.

Courchesne WE, Ozturk S. 2003. Amiodarone induces a caffeine-inhibited, MID-1-dependent rise in free cytoplasmic calcium in Saccharomyces cerevisiae. Mol Microbiol, 47:223-34.

Courchesne WE. 2002. Characterization of a novel, broad-based fungicidal activity for the antiarrhytmic drug amiodarone. J Pharmacol Exp Ther. 300:195-9.

Croft SL, Barrett MP, Urbina JA. 2005. Chemotherapy of trypanosomiases and leishmaniasis. Trends Parasitol, 21:508-12.

Dias E, Laranja FS, Miranda A, et al. 1956. Chagas' disease; a clinical, epidemiologic, and pathologic study. Circulation, 14:1035-60.

Gupta SS, Ton VK, Beaudry V, et al. 2003. Antifungal activity of amiodarone is mediated by disruption of calcium homeostasis. J Biol Chem, 278:28831-9.

Lawn SD, Armstrong M, Chilton D, et al. 2006. Electrocardiographic and biochemical adverse effects of sodium stibogluconate during treatment of cutaneous and mucosal leishmaniasis among returned travellers. Trans R Soc Trop Med Hyg, 100:264-9.

Marin-Neto JA, Cunha-Neto E, Maciel BC, et al. 2007. Pathogenesis of chronic Chagas heart disease. Circulation, 115:1109-23.

Mendes DG, Lauria-Pires L, Nitz N, et al. 2007. Exposure to mixed asymptomatic infections with Trypanosoma cruzi, Leishmania braziliensis and Leishmania chagasi in the human population of the greater amazon. Trop Med Int Health, 12:629-36.

Murray HW, Berman JD, Davies CR, et al. 2005. Advances in leishmaniasis. Lancet, 366:1561-77.

Opie LH, Gersh BJ. 2005. Antiarrhytmic drugs and strategies. In: Drugs for the Heart. 6th edition. Philadelphia: Elsevier Inc., 218-74.

Schwartz E, Hatz C, Blum J. 2006. New world cutaneous leishmaniasis in travellers. Lancet Infect Dis, 6:342-9.

Seear MD. 2000. Manual of tropical pediatrics. Cambridge: Cambridge University Press, Inc.

Silveira FT, Lainson R, Corbett CEP. 2004. Clinical and immunopathological spectrum of American cutaneous leishmaniasis with special reference to the disease in Amazonian Brazil-A review. 99:239-51.

Singh BN. 2006. Amiodarone: a multifaceted antiarrhythmic drug. Curr Cardiol Rep, 8:349-55.

Urbina JA, Docampo R. 2003. Specific chemotherapy of Chagas disease: controversies and advances. Trends Parasitol, 19:495-501. 